\title{
Community Driven Digital Signal Processing Laboratories in Connexions
}

\author{
Richard Baraniuk, ${ }^{\circ}$ Hyeokho Choi, ${ }^{\circ}$ Douglas L. Jones, ${ }^{\diamond}$ Lee Potter ${ }^{\dagger} *$ \\ Department of Electrical and Computer Engineering \\ $\diamond$ University of Illinois at Urbana-Champaign \\ ${ }^{\circ}$ Rice University, ${ }^{\dagger}$ Ohio State University
}

\begin{abstract}
The conventional textbook is largely inadequate for digital signal processing (DSP) laboratory education due to inherent factors such as a small and fragmented market and rapid hardware obsolescence. Freely available open-content materials that enable and promote both local customization and further development by a community of educators offers a fresh approach to lab text development that can surmount these barriers. In this paper, we overview a joint effort organized by the Connexions Project to develop a large pool of DSP lab modules sufficient to serve as the complete, stand-alone text for several types of DSP lab courses.
\end{abstract}

\section{Introduction}

Digital signal processing (DSP) laboratory courses are difficult to serve with conventional commercial textbooks. First, the market is relatively small, thus precluding a major investment by authors and publishers in the hope of a substantial commercial success. Second, the market is fragmented; different lab equipment or DSP microprocessors are used at each educational institution, and the course level, structure, and content may differ greatly, thus necessitating different textbooks or versions to serve an already small market. Essential materials generally include tutorials on using the particular lab setup and equipment and code examples or wrappers that are site-specific. Third, DSP hardware changes quite rapidly while the development and publication of a printed textbook takes quite some time, thus making a text nearly obsolete at the time of publication. For these reasons, conventional commercial textbooks and publishers are unlikely to ever serve DSP lab courses

*Web: cnx.rice.edu; Email: \{richb,choi\}@ rice.edu,dl-jones@uiuc.edu, potter@ee.eng.ohio-state.edu

Proceedings of the 2004 American Society for Engineering Education Annual Conference \& Exposition Copyright (C)2004, American Society for Engineering Education 
effectively. A new approach is needed to provide high-quality materials for such courses in a timeand cost-effective manner.

While the limitations of conventional textbooks preclude them from effectively serving DSP lab courses, the situation is not hopeless. Most courses share many commonalities; for example, almost every DSP lab course covers FIR and IIR filter implementation. However, certain details, such as the assembly language instructions for a particular microprocessor, differ between platforms and preclude the use of a common printed text. Relatively minor modifications of the materials could effectively exploit the commonality of the bulk of the material but are currently not possible due to potential copyright violations and the fixed print medium.

The open-source software movement (Linux and GNU, for example) suggests a new paradigm that could overcome these limitations. In this spirit, we have undertaken a major community-based project to develop open instructional materials for DSP lab education within the framework of Connexions $[1,2]$. A complete set of materials sufficient to teach a signal processing lab is now available, and the materials have been successfully used each semester since the fall of 2002 as the sole text for a large DSP lab at the University of Illinois at Urbana-Champaign (UIUC). Collaborative development continues to expand these materials so that they will soon be in use at multiple universities. Being open, they are freely available to all educators and users for modification, extension, and use.

\section{Overview of the Connexions project}

Connexions is a collaborative, community-driven approach to authoring, teaching, and learning that aims both to convey the dynamic continuum of knowledge and to ease and speed the course and curriculum development process. Launched in 1999 at Rice University, its hallmarks include:

- a Content Commons of diverse educational materials that are modularized for easy reuse and available free-of-charge to anyone in the world under an open-content license;

- rapid, collaborative authoring of the materials by global communities of authors;

- high-quality materials, thanks to an iterative development process and an inherent quality assessment mechanism;

- flexible, dynamic construction of customized courses and curricula, enabled by a coherent format (XML) and delivered in a variety of forms, from web pages to e-books to paper texts;

- visualization and navigation of the "connexions" among concepts, courses, and curricula.

See [1] and cnx.rice. edu for more information.

Connexions can be viewed as a kind of "course factory" (see Figure 1). A global community of authors, using special authoring tools (top), continually converts "raw knowledge" into small,

Proceedings of the 2004 American Society for Engineering Education Annual Conference \& Exposition

Copyright (c) 2004, American Society for Engineering Education 


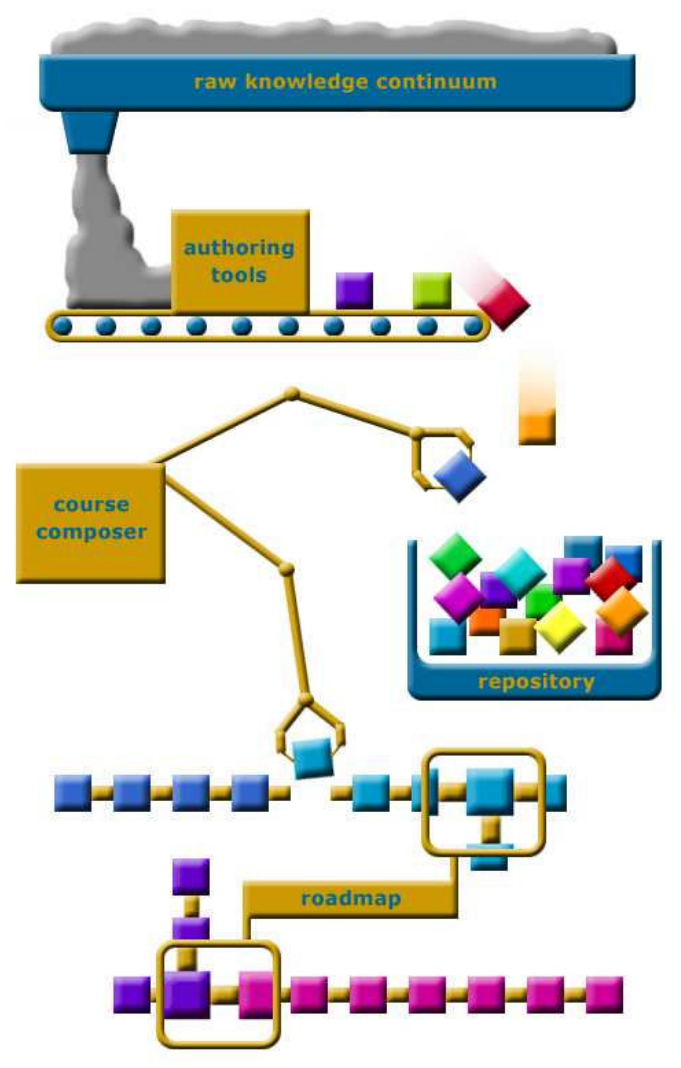

Figure 1: Connexions as a "course factory."

self-contained modules of information and places them in the Content Commons repository (middle right) to be used, re-used, updated, and adapted. (Modules can be thought of as special web pages that can contain hyperlinks, text, equations, applets, simulations, videos, and other multimedia elements.) Instructors use a Course Composer software tool (middle left) to weave modules into customized courses. Students and other learners view modules and courses using special visualization and navigational tools (bottom) designed to highlight the non-linear connexions among concepts.

The Connexions architecture and software toolkit consists of an XML language for content markup (including mathML for mathematics), a database repository to store and retrieve the modules, the Course Composer to build courses, an annotation capability for instructors to customize modules and for students to personalize them, a Roadmap browser to navigate courses and modules, and a printing tool that converts a course into a ready-to-print pdf text complete with table of contents and index. An integrated XML/mathML document editor is planned for the near future, as are wizards to help convert from $\mathrm{LT}_{\mathrm{E}} \mathrm{X}$, Microsoft Word, and other formats into XML. On the web, Connexions modules are viewable with a range of browsers, including Mozilla, Netscape

Proceedings of the 2004 American Society for Engineering Education Annual Conference \& Exposition Copyright (C)2004, American Society for Engineering Education 
6 and above, and Microsoft Internet Explorer. The Roadmap navigator currently prefers Mozilla and Netscape. (Safari and Opera support are on the way.) Last but not least, the Connexions author website enables groups of authors to form ad hoc workgroups to collaboratively develop new modules and courses. All Connexions software and tools are distributed open-source and freeof-charge. Release 1.0 versions are in live use by faculty at Rice University, UIUC, Ohio State University (OSU), and a number of other institutions (see cnx.rice. edu for more information).

Rather than the traditional one-author-to-one-textbook content development model, Connexions links worldwide communities of authors to collaboratively create, expand, revise, and maintain the Content Commons. All materials are freely available under a Creative Commons opencontent license analogous to the general public license (GPL) used for GNU and Linux software (see creativecommons.org for more information). Anyone can copy, modify, and redistribute modules, most even for commercial use. The result is a dynamic, up-to-date content base that makes the latest knowledge globally available.

Modularity and open-content development substantially lowers the barrier to entry into the author community. Most faculty currently do not write textbooks due the large time commitment required. Since in Connexions authors can now contribute a high-quality, high-impact module in an evening or weekend, many more college faculty, industry professionals, K-12 teachers, and even talented students will contribute materials. Consider this quote from an electrical engineering faculty member: "For years I have wanted to write a textbook, because I love to write about FFTs. However, any complete text in my field also has to cover $z$-transforms, on which I have no interest in writing." Connexions will allow this faculty member to contribute his (excellent) FFT material and then weave a custom text for his course using contributions from other authors passionate about $z$-transforms.

In February 2004, Connexions hosts over 1,650 modules in the Content Commons and is used as the primary text for 35 courses. A particular content focus is DSP, manifested in two projects: graduate-level DSP theory materials [1], and DSP labs [2], the subject of this paper.

\section{The case for Connexions DSP lab texts}

Connexions provides a near-ideal venue for developing DSP lab texts (or indeed any type of lab text). The low volume market has never been of great commercial interest, so the free, open-content model breaks the low-volume/high-cost barrier arising in print-based commercial publishing. The variety of different processors and lab setups makes it nearly impossible for a single printed text to address the needs of most laboratories, but the natural division of such courses into theory- and application-oriented concepts allows for the reuse of most materials across lab courses, with the exception of only the most platform-specific topics.

For example (see Figure 2), an assignment revolving around FIR filtering will typically involve a theory section on the concept, simulation exercises (in an environment such as Matlab), discus-

Proceedings of the 2004 American Society for Engineering Education Annual Conference \& Exposition Copyright (C)2004, American Society for Engineering Education 


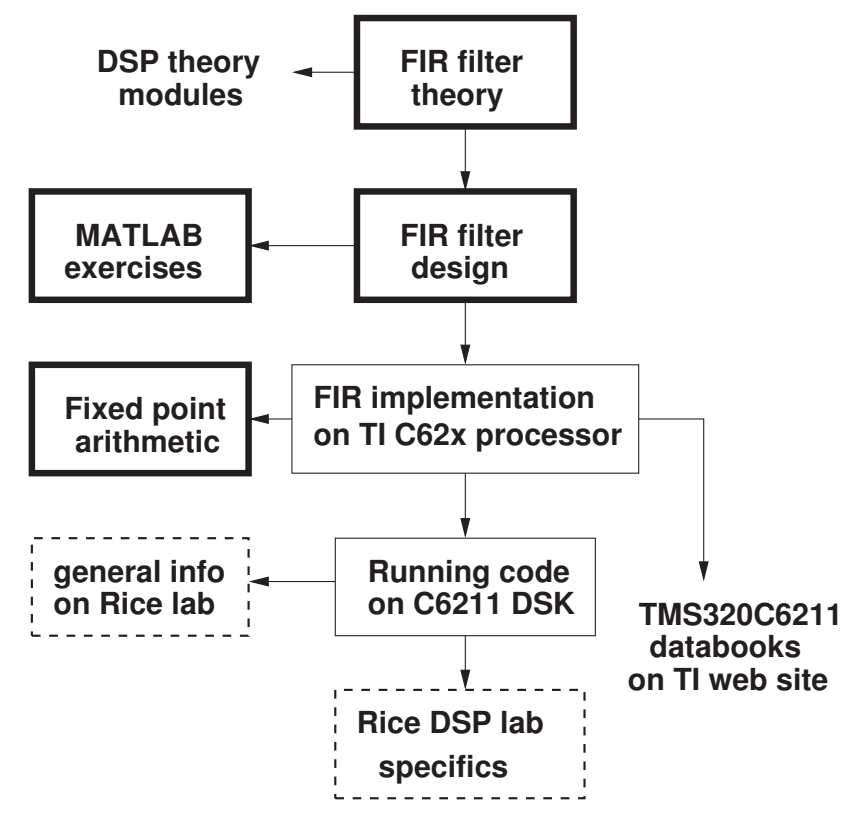

Figure 2: Connexions FIR filtering lab as used in Rice University ELEC 434. Each box represents a module, and arrows represent hyperlinks between modules. Boxes with thick boundaries indicate DSP theory and exercise modules that can be used at other institutions with little modification. Boxes with thin boundaries are processor-specific. Boxes with dashed boundaries are specific to a particular equipment setup.

sions of generic hardware theory (fixed-point arithmetic, for example) and specific hardware theory (FIR filter algorithms in Texas Instruments TMS320C6211 assembly language, for example), and finally a section discussing the implementation and testing that are specific to a particular lab platform. The theory modules are readily available in Connexions and have most likely been seen by the students in their other DSP courses if those are Connexions-based. In fact, using already familiar DSP theory modules as a jumping-off point will allow the students to not only establish greater continuity with their other course work but also begin the lab from comfortable territory. Simulation modules can be found both in DSP theory courses and other lab courses (even those involving just Matlab exercises). Hardware theory modules can be found similarly in appropriate courses.

Thus, in a fully developed Connexions environment, an instructor wishing to build a DSP lab class need only choose and assemble a set of pre-existing modules to craft the majority of her class. The remainder of the class can then be written in module form to cover the actual implementation issues of the local hardware platform. Even at this stage, she can potentially leverage modules provided by the equipment manufacturer (such as modules covering the assembly language instruction set for the microprocessor being used). A handful of segue modules (either written by the instructor or borrowed from similar DSP labs) tying theory to implementation can round out the course. This, of course, represents the minimum amount of effort required to craft a quality DSP lab. The instructor is free, under the open-content license, to customize and improve 
existing modules as she sees fit. These, in turn, may gain popularity and become the material most often used in other DSP labs, as commonly occurs in such an open-source development environment.

In short, though instructors may lack the time, resources, or desire to independently develop a complete text of lab materials, they can still fashion, with relatively little effort, a comprehensive DSP lab text that accommodates the hardware available at their respective institutions. By leveraging pre-existing materials and perhaps adding a module or two on topics of particular interest and expertise, an instructor can create, distribute, and share a full lab text with little more effort than that required for preparing supplementary handouts with assignments and site-specific information.

\section{A tale of three DSP Labs}

UIUC ECE 320. The Electrical and Computer Engineering department at UIUC has offered a senior-level DSP lab course (www. ews.uiuc.edu/ ece320) since 1989. Due to the large demand for the course, it is now offered in both the Fall and Spring semesters with a typical enrollment of 40-50 students each semester. For its first four years, the course used the TI TMS32010 DSP microprocessor and an accompanying textbook coauthored by the instructor [3]. Obsolescence of the microprocessor led to a shift to the Motorola DSP56000 family for the next six years, for which an excellent text was initially available [4]. However, as the hardware was upgraded every two or three years and as the lab assignments evolved, it became necessary to develop more and more supplemental material (in $\mathrm{LT}_{\mathrm{E}} \mathrm{X}$ ) to address the differences between the text and the hardware and software environment and to introduce topics not covered in the text. Eventually, these materials entirely replaced the commercial textbook. When the course migrated to the Texas Instruments TMS320C549-based platform, no suitable text was available, so UIUC modified the text materials for this new hardware and software environment. The materials have always been openly available on the course website, and have been used at other institutions (such as the University of Washington) as a basis for developing their own labs.

UIUC ECE 320 is a semester-long project-oriented DSP lab [5]. It consists of roughly two equal parts: a series of weekly lab assignments, including an introduction to the TI TMS320C549 and its DSP development environment, real-time FIR, IIR, and multirate filtering, spectral analysis using the FFT, and a digital communications transmitter. Students work together in pairs on these lab assignments and are orally quizzed individually after completing each assignment. The materials for each week are semi-self-paced and typically consist of a brief review of the relevant signal processing concepts, a design or familiarization exercise (often Matlab-based), and a realtime implementation assignment using the TMS320C549. After completing these common weekly assignments in mid-semester, student teams conceive a substantial real-time DSP project of their choice and spend the remainder of the semester designing, simulating, implementing, and testing it. Supplementary modules introducing students to the basics of digital communication, adaptive filtering, speech processing, and audio signal processing accelerate students' progress on projects in these areas.

Proceedings of the 2004 American Society for Engineering Education Annual Conference \& Exposition Copyright (c)2004, American Society for Engineering Education 
Rice ELEC 434. The Rice University Electrical and Computer Engineering department offers a senior-level DSP lab course (www.dsp.rice.edu/courses/elec434) based on the TI TMS320C6211 DSP processor. When the course was first offered in Fall 2000 there were no textbooks available for this processor, and so Hyeokho Choi developed a text from scratch. The initial set of notes adapted the UIUC ECE 320 lab materials to the new processor (in LTEX). Over the last two years, Rice has added more material covering the C6x processor architecture and C6x assembly language programming for students less familiar with microprocessor programming. In addition, since a DSP theory course is not a prerequisite to ELEC 434, the basic theory necessary for the lab experiments (such as filter design) is taught in the lecture sessions.

OSU EE 609. The Ohio State University DSP lab (eewww.eng.ohio-state.edu/ potter/EE 609) introduces real-time applications using a fixed-point DSP microprocessor, the Motorola DSP56002. The quarter-long lab consists of six structured labs and a mini-project. Some familiarity with microprocessor architectures, assembly language programming, and discrete-time signal processing is assumed from the required undergraduate curriculum.

\section{Connexions DSP labs}

In 2001 we realized that Connexions could provide a new and better mechanism for sharing DSP lab materials and for enabling a community of authors to collaboratively develop a common instructional resource of ever greater breadth and utility. Former UIUC ECE 320 teaching assistants and instructors and the Connexions staff at Rice University developed a complete set of lab modules in Connexions based on the original ECE 320 materials. In fact, two versions of ECE 320 were developed, one all-assembly and one assembly-with-C-compiler. The current complete course in pdf format is available at (www. ews. uiuc.edu/ ece320); the courses and individual modules are available in the Connexions Content Commons (cnx.rice.edu).

To confirm the capability of the system and the efficacy and completeness of the materials, ECE 320 (physically located in Urbana, Illinois) was taught during the Fall 2002 semester using solely Connexions-based materials located on the Connexions server (physically located at Rice University in Houston, Texas). The UIUC staff found it easier to maintain and update their materials in Connexions than on their own website, which was a pleasant surprise.

To augment the UIUC ECE 320 materials for Rice ELEC 434, a series of modules are being developed to cover both basic DSP theory and TMS320C6211 based algorithm implementations. Figure 2 illustrates an example FIR filtering lab developed using Connexions modules. In the figure, each box represents a different module, which were either obtained from other Connexions courses (for example, Rice ELEC 301, Signals and Systems) or written from scratch. The boxes with thick boundaries indicate the DSP theory and exercise modules that can be used at other institutions with little modification, since they are common to any FIR filtering lab. The boxes with thin boundaries are the processor-specific parts of the lab. For example, one can replace them with modules developed for the TMS320C549 processor used at UIUC. The boxes with dashed

Proceedings of the 2004 American Society for Engineering Education Annual Conference \& Exposition Copyright (C)2004, American Society for Engineering Education 
boundaries are specific to the Rice lab setup and would need to be replaced with institution-specific lab setup information.

For OSU EE 609, the modular design of the lab exercises and related DSP tutorial materials allows each quarter's instructor to easily customize the application examples - such as echo location and OFDM signaling. Further, modules have been inserted to cover finite-precision issues not covered in a quarter-long DSP theory course. Finally, supplementary modules from the Connexions Content Commons provide resource material for students to successfully complete a mini-project of their choosing within the abbreviated quarter schedule.

\section{Using the Connexions DSP labs}

The Connexions DSP lab materials build on over fourteen years of DSP lab instruction and over ten years of collaborative development of instructional lab materials at UIUC, Rice, and OSU. Much of the content has evolved in tandem with the UIUC ECE 320 course, and to a large extent reflects its goals and structure. With the addition of the Rice ELEC 434 and OSU EE 609 modules, the material is suited for a variety of course organizations, including

- a semester-long project-oriented DSP lab,

- a quarter- or semester-long DSP lab,

- a hands-on lab supplement as part of a signal processing theory course,

- a self-study course in real-time DSP implementation.

Being highly modular, the connexions modules can be easily modified and regrouped to develop texts to serve various different goals. A course emphasizing DSP techniques might forgo a major project and instead use the supplementary modules to complete a quarter or semester of weekly lab assignments. A one-hour hands-on lab supplement to a signal-processing lecture course could stretch the first few units (through spectral analysis, for example) over a semester, thereby reinforcing and enhancing students' understanding of the core signal processing theory and algorithms. Due to the self-paced, tutorial design of the materials, a student can independently learn the aspects of real-time DSP implementation that interest them, for example as preparation for an independent design project.

The UIUC, Rice, and OSU materials and assignments reflect our belief that a thorough instruction in signal processing implementation requires exposure to assembly-language programming of fixed-point DSP microprocessors, as this represents an important component of current and at least near-future industrial practice. Instructors with other goals or perspectives may find most of the tutorial, design material, and assignments relevant even if they choose compiler-based or non-real-time implementation.

Proceedings of the 2004 American Society for Engineering Education Annual Conference \& Exposition Copyright (C)2004, American Society for Engineering Education 
Laboratories using different development systems or different DSP microprocessors will likely find the material well suited for their needs; only the hardware-specific instructions need be modified. By choosing different sets of Connexions modules and modifying and rewriting the hardwarespecific ones, many different lab texts supporting different lab setups can be easily generated with minimal effort.

All of these Connexions courses and modules are available for free use and re-use at cnx.rice.edu.

\section{Conclusions}

Due to inherent factors such as a small and fragmented market and rapid hardware obsolescence, the conventional textbook is inadequate for supporting DSP lab education. Copyright restrictions and the printed textbook format prevent site-specific modification and sharing of textual resources that could enable the time- and cost-effective development of materials specialized for each course. Freely available open-content materials that allow such modification and further development by a community of educators offers a fresh approach to lab text development that can surmount these barriers.

A joint effort under the aegis of the Connexions project to develop a pool of DSP lab modules has resulted in a large body of material sufficient to serve as the complete, stand-alone text for several types of DSP lab courses. The materials include the complete texts for two versions of the UIUC ECE 320 DSP lab course (TI TMS320C549-based all-assembly and assembly-with-Ccompiler) and the Rice ELEC 434 course (TI TMS320C6211-based). ECE 320 has been taught successfully in the Fall 2002 semester served solely from the Connexions website. No appreciable difficulties or lack of availability were experienced in doing so. The materials are open-content licensed, so anyone is free to use, modify, and add to them.

Connexions provides an excellent framework for the development and provisioning of open DSP lab materials. However, our initial experience in using Connexions for this purpose suggests many enhancements that could improve its utility for lab instruction. Convenient support of sitespecific tags or parameters, such as allowing the local directory path to the assembler executable or the link to the course web page to be defined without editing of every module, would substantially reduce the effort required to specialize materials for an individual lab. The ability to interface directly with software or hardware, such as Matlab or an oscilloscope, could enable new kinds of multimodal instruction. Finally, the capability to support on-line exercises for the students would promote active learning with instant feedback, thus accelerating students' progress. The development of these features will be greatly aided by the open-source nature of Connexions' architecture and software.

Proceedings of the 2004 American Society for Engineering Education Annual Conference \& Exposition Copyright (C)2004, American Society for Engineering Education 


\section{Acknowledgements}

Thanks to the Hewlett Foundation, CLASS Foundation, and Rice University for supporting the development, launch, and growth of Connexions. Thanks to Texas Instruments and Motorola for the DSP hardware and software development tools. And thanks to the dedicated Connexions staff, including Ricky Radelli-Sanchez, Adan Galvan, and Brent Hendricks.

\section{References}

[1] R. G. Baraniuk, C. S. Burrus, B. Hendricks, G. Henry, A. Hero, D. Johnson, D. L. Jones, J. Kusuma, R. Nowak, J. Odegard, L. C. Potter, and K. Ramchandran, "Connexions: DSP education for a networked world," in Proc. IEEE Int. Conf. Acoustics, Speech, and Signal Processing - ICASSP'02, Orlando, FL, May 2002.

[2] S. Appadwedula et al., "Open-content signal processing laboratories in Connexions," in Proc. IEEE Int. Conf. Acoustics, Speech, and Signal Processing - ICASSP'03, Hong Kong, 2003.

[3] D. L. Jones and T. W. Parks, A Digital Signal Processing Laboratory for the TMS32010, Prentice-Hall, Englewood Cliffs, NJ, 1988.

[4] M. El-Sharkawy, Digital Signal Processing Applications with Motorola's DSP56000 Processor, Prentice-Hall, Englewood Cliffs, NJ, 1997.

[5] D. L. Jones, "Designing effective DSP laboratory courses," in Proc. IEEE Int. Conf. Acoustics, Speech, and Signal Processing - ICASSP'01, Salt Lake City, UT, May 2001. 